Article

\title{
Limited Effects of Low-to-Moderate Aerobic Exercise on the Gut Microbiota of Mice Subjected to a High-Fat Diet
}

\author{
Filipe M. Ribeiro ${ }^{1,2,3}$, Camila F. A. Ribeiro ${ }^{4}$, Ana C. M. Garcia ${ }^{3}$, Alinne P. Castro ${ }^{4}$, \\ Jeeser A. Almeida ${ }^{5}$, Octavio L. Franco ${ }^{2,4}$ and Bernardo A. Petriz ${ }^{2,3, *(D)}$ \\ 1 Post-Graduation Program in Physical Education, Catholic University of Brasilia, \\ Brasilia 71966-700, DF, Brazil; filipemouraudf@gmail.com \\ 2 Center for Proteomic and Biochemical Analysis, Post-Graduation in Genomic and Biotechnology Sciences, \\ Catholic University of Brasilia, Brasília 71966-700, DF, Brazil; ocfranco@gmail.com \\ 3 University Center-UDF, Research Group of Molecular Exercise Physiology, Brasilia 70390-045, DF, Brazil; \\ anacaiana@gmail.com \\ 4 S-Inova Biotech, Catholic University Dom Bosco, Biotechnology Program, \\ Campo Grande, 79000-000, MS, Brazil; camila.faribeiro@gmail.com (C.F.A.R.); palinne@gmail.com (A.P.C.) \\ 5 Programa de Pós-Graduação em Saúde e Desenvolvimento na Região Centro Oeste-PPGSD, \\ Faculdade de Medicina-FAMED, Universidade Federal de Mato Grosso do Sul, \\ Campo Grande 71966-700, MS, Brazil; jeeser@gmail.com \\ * Correspondence: bernardopetriz@gmail.com; Tel.: +55-061-98644-1081
}

Received: 6 December 2018; Accepted: 3 January 2019; Published: 11 January 2019

check for updates

\begin{abstract}
Several studies have indicated that diet and exercise may modulate the gut microbiota in obese subjects. Both interventions were shown to alter the microbiota orthogonally. However, this relationship has not been fully explored. This study analyzed the effects of low-to-moderate aerobic training on the fecal microbiota of mice subjected to a high-fat diet (HFD). Here, 40 male mice (C57Bl/6) were divided into two groups with standard diet (SD; $12.4 \%$ lipid) and HFD (60.3\% lipid) for four months. These groups were divided into four, named SD control, HF control, SD trained and $\mathrm{HF}$ trained. All animals were submitted to an incremental test to estimate low-to-moderate maximum speed. Training consisted of $30 \mathrm{~min} \cdot \mathrm{day}^{-1}, 5$ days/week, for 8 weeks. The HFD increased the body weight $(p<0.0001)$ and adiposity index $(p<0.05)$. HFD also negatively influenced performance in exercise training. Moreover, the diversity of gut microbiota was reduced by the HFD in all groups. A low-to-moderate exercise was ineffective in modulating the gut microbiota composition in mice subjected to HFD. These findings suggest that two months of low-to-moderate exercise does not achieve a preponderant modulatory effect on shaping microbiota when submitted to the high-fat diet.
\end{abstract}

Keywords: gut microbiota; obesity; low-to-moderate intensity; physical activity; diet

\section{Introduction}

Obesity is one of the most impactful chronic nontransmissible diseases in the world [1]. To date, about $13 \%$ of adults are obese, and $39 \%$ are considered to be overweight worldwide [2]. Also, the obesity pandemic has resulted in high public health expenditure [3] and a high death rate worldwide [4]. Due to the high pathogenesis complexity, significant efforts are being made by public health policies and scientific research to establish novel strategies in its prevention and treatment [5].

Studies have shown that the gut microbiota plays a significant role in the pathogenesis of obesity [6], where the positive regulation within its content may be used as a clinical treatment [7-9]. The gut microbiota consists of millions of microorganisms responsible for a series of physiologic 
functions related to the maintenance of host homeostases, such as immunologic maturation within the gut, digestion of complex polysaccharides, and protection against pathogens [6,10]. Diverse factors such as host-genotype interaction, environmental factors [11], pathogens [12], antibiotics [13] and lifestyle [14] are also known to shape the gut microbiome. However, Western diets, marked by high caloric values and high fat content, are one of the well-known factors in the induction of obesity [15]. Lately, a series of studies have shown that these obesogenic diets also modulate the gut microbiota, leading to a specific microbiota profile, with less diversity, thus with greater facility in harvesting energy from digestion and accumulating fat [15]. Interestingly, the transplantation of fecal microbiota from lean subjects to obese individuals seems to be effective in enhancing its diversity and composition [16]. In this context, the modulation of the gut microbial consortia is being investigated as a possible novel strategy in the treatment of obesity.

Furthermore, and in parallel with dietary manipulation, physical activity was also shown to induce alterations within the composition, abundance and gut microbiota diversity in experimental models [17] and humans [18,19]. To this end, a series of exercise stimuli have been used to assess the role of exercise in the modification of the gut consortia, which include high- [20] and moderate-intensity training [21,22], and a comparison between voluntary and forced exercise [23]. This last study indicated that both stimuli distinctively alter the gut bacteria, which must be considered for further interpretations. Nevertheless, only a few studies have investigated the role of exercise and dietary intervention in obese subjects $[8,24]$. Taking these facts into consideration, the shaping of the gut microbiota through exercise may be one alternative strategy for the prevention and treatment of obesity $[10,25]$. However, there are no studies about the effects of low-to-moderate exercise on gut microbiota, even less when associated with the previous induction of a high-fat diet (HFD).

Both the gut microbiota and exercise have been implicated in obesity $[10,26]$. However, the effect of controlled low-to-moderate exercise training on gut microbiota of mice that were induced by an HFD remains unexplored. For this reason, in the present study, the composition of gut microbiota and the training intervention were evaluated using the $16 \mathrm{~S}$ rDNA gene sequence from fecal samples. Due to the growing research into the effects of exercise training on gut microbiota of obese subjects $[17,27]$, we hypothesized that a protocol of eight months of exercise training performed at a low-to-moderate intensity (50\% of maximal velocity) would play a significant role in gut microbiota modulation by HFD induction. Our results, described below, show the limited effects of exercise on the obesity context by altering gut microbiota.

\section{Material and Methods}

\subsection{Animals}

Animals were obtained from the Bioassays Laboratory of the Catholic University of Brasilia (UCB), and all animals (40 isogenic male mice, C57BL6) started the experiment at $\sim 4$ weeks of age. The mice were individually allocated in separate cages to avoid the "cage effect" (animals kept in the same environment tend to have similarities in their microbiota) [28]. The bioterium temperature was maintained at $23^{\circ} \mathrm{C}\left( \pm 2{ }^{\circ} \mathrm{C}\right)$ and kept in a $12 \mathrm{~h}$ light-dark cycle environment. The Animal Use Ethics Committee (CEUA) of the Catholic University of Brasilia, Brazil, approved the methods and conduct that were used in the experiment (UCBDOC, no. 026/15). After experimental procedures, animals were anesthetized with $2 \%$ xylazine $\left(50 \mathrm{mg} \cdot \mathrm{kg}^{-1}\right)$ and $10 \%$ ketamine $\left(80 \mathrm{mg} \cdot \mathrm{kg}^{-1}\right)$ and euthanized by cervical dislocation. All procedures were performed following the relevant guidelines and regulations as here described. Through the entire experiment, all efforts were made to minimize animal suffering, and a veterinarian, and a technician specialized in Confined Animal Facilities, accompanied all the procedures described below. 


\subsection{Experimental Design and Exercise Training}

Right after weaning, the 40 animals were divided into two groups: a group fed with a standard $\operatorname{diet}(\mathrm{SD}, 68.8 \% \mathrm{kcal}$ as carbohydrate, $18.8 \% \mathrm{kcal}$ as protein and $12.4 \% \mathrm{kcal}$ as lipids) $(n=20)$ and a group fed with a high-fat diet (PRAGSOLUÇÕES Biociências, BRA), (HFD, 21.3\% kcal as carbohydrate, $18.4 \% \mathrm{kcal}$ as protein and $60.3 \% \mathrm{kcal}$ as fat, $n=20$ ) as described by Cano, Santacruz [7]. The objective of this process is to induce chronic obesity in approximately 4 months of feeding on the HFD [29]. All animals received water ad libitum throughout the experiment. The animals' body weight, as well as the weekly intake (in grams), were weighed by an analytical balance (Shimadzu, AUY220) throughout the experiment. Also, the caloric value of the weekly intake was calculated by the value in grams ingested per week (g/week). Furthermore, after euthanasia, the adipose tissue was collected, and total body fat was measured by the calculation of the adiposity index (AI \%; the sum of the weight of retroperitoneal, subcutaneous epididymal and omental white adipose tissue/body weight $\times 100$ ) [30].

After 4 months of diet-induced obesity, and before exercise training, all animals were submitted to an initial acclimatization and adaptation process in the environment where the training took place. The treadmill (Treadmill Exer 3/6, Columbus Instruments, Columbus, OH, USA) used can force exercise by a mild electronic shock grid $(0.2 \mathrm{~mA})$ at the rear of the treadmill. The acclimatization processes occurred on the treadmill with no movement from 10 to $30 \mathrm{~min}$ for five days. After that, the animals were adapted for three weeks with time and velocity being increased up to the limit of $20 \mathrm{~m} \cdot \mathrm{min}^{-1}$, as described by Almeida et al. [31,32]. After the adaptation period, the mice were randomly distributed into four groups: standard diet control (SD-C, $n=10$ ); standard diet trained (SD-T, $n=10$ ); high-fat control (HF-C, $n=1$ ); and high-fat trained (HF-T, $n=10$ ) (Figure 1). An incremental test of maximum velocity (IT- $\mathrm{V}_{\max }$ ) was performed in three periods: before training, after 4 weeks of training and after the end of the training protocol (total of eight weeks of training). The $\mathrm{V}_{\max }$ was performed on a treadmill running with the start of the test at $6 \mathrm{~m} \cdot \mathrm{min}^{-1}$ and with increments of $3 \mathrm{~m} \cdot \mathrm{min}^{-1}$ every three minutes until exhaustion of the animal. The objective of this test consists of the evaluation of the $\mathrm{V}_{\max }$ and how the evaluation was individualized. All animals performed the IT- $\mathrm{V}_{\max }$. After an evaluation of the $\mathrm{V}_{\max }$, the animals trained for 2 months at an intensity relative to $50 \%$ of the $V_{\max }$, five times a week with duration of $30 \mathrm{~min}$ per session (Figure 1). Control groups remained untrained for 8 weeks; however, these groups ran the IT- $\mathrm{V}_{\max }$.

\subsection{Fecal DNA Extraction and $16 S$ rDNA Gene Sequencing}

Three fecal samples of each animal were collected during the following periods: pre-diet (absolute control), before training and after 8 weeks of training. The fecal collection was performed $24 \mathrm{~h}$ after the last bout of the exercise session. Samples were immediately stored in RNAlater ${ }^{\circledR}$ solution (Life Technologies, Carlsbad, CA, USA) until stored at $-80^{\circ} \mathrm{C}$. Fecal microbial DNA was extracted from $\sim 0.25 \mathrm{~g}$ using the PowerFecal DNA Isolation Kit (MoBio, Carlsbad, CA, USA) according to the manufacturer's instructions. The triplicate DNA extractions were not pooled together, being analyzed individually. Hybridization of the conserved region of the $16 \mathrm{~S}$ rDNA gene occurred with the use of the regions V3 (5'-CCTACGGGNGGCWGCAG-3') to V4 (5'-TACHVGGTATCTAATCC- $\left.3^{\prime}\right)$ to the bacterial domain, generating a fragment with $\sim 444 \mathrm{bp}$. However, for these fragments to be compatible with the MiSeq Illumina sequencing platform, specific adapters were coupled: 5'-TCGTCGGCAGCG TCAGATGTGTATAAGAGACAG-3' (V3F) and 5'-GTCTCGTGGGCTCGGAG ATGTGTATAAGAGACAGGAC-3' (V4R). Fragments of the $16 \mathrm{~S}$ rDNA genes were amplified via polymerase chain reaction (PCR), in this order: microbial DNA $\left(5 \mathrm{ng} \cdot \mu \mathrm{L}^{-1}\right)$, amplicon PCR Primer $1 \mu \mathrm{M}$, amplicon PCR Reverse Primer $1 \mu \mathrm{M}$ and 2× KAPA HiFi HotStart ReadyMix with a final reaction volume of $25 \mu \mathrm{L}$. The reaction cycle was carried out with an initial denaturation of $3 \mathrm{~min}$ at $94{ }^{\circ} \mathrm{C}$, followed by 35 cycles with denaturation for $30 \mathrm{~s}$ at $94{ }^{\circ} \mathrm{C}$, annealing for $60 \mathrm{~s}$ at $50^{\circ} \mathrm{C}$ and last, extension for $90 \mathrm{~s}$ at $72{ }^{\circ} \mathrm{C}$, followed by a final extension from $7 \mathrm{~min}$ to $72{ }^{\circ} \mathrm{C}$ and cooling to $10^{\circ} \mathrm{C}$. Subsequently, the PCR products were quantified via Qubit ${ }^{\circledR}$ (Life Technologies) and then sent directly to BPI Genotyping-Botucatu/SP, for the preparation of the amplicons, such as the attachment of 
the barcodes with the Nextera ${ }^{\circledR}$ XT Index Kit and running the run on the MiSeq Illumina platform, according to the standard protocol (16S Metagenomic Sequencing Library Preparation).

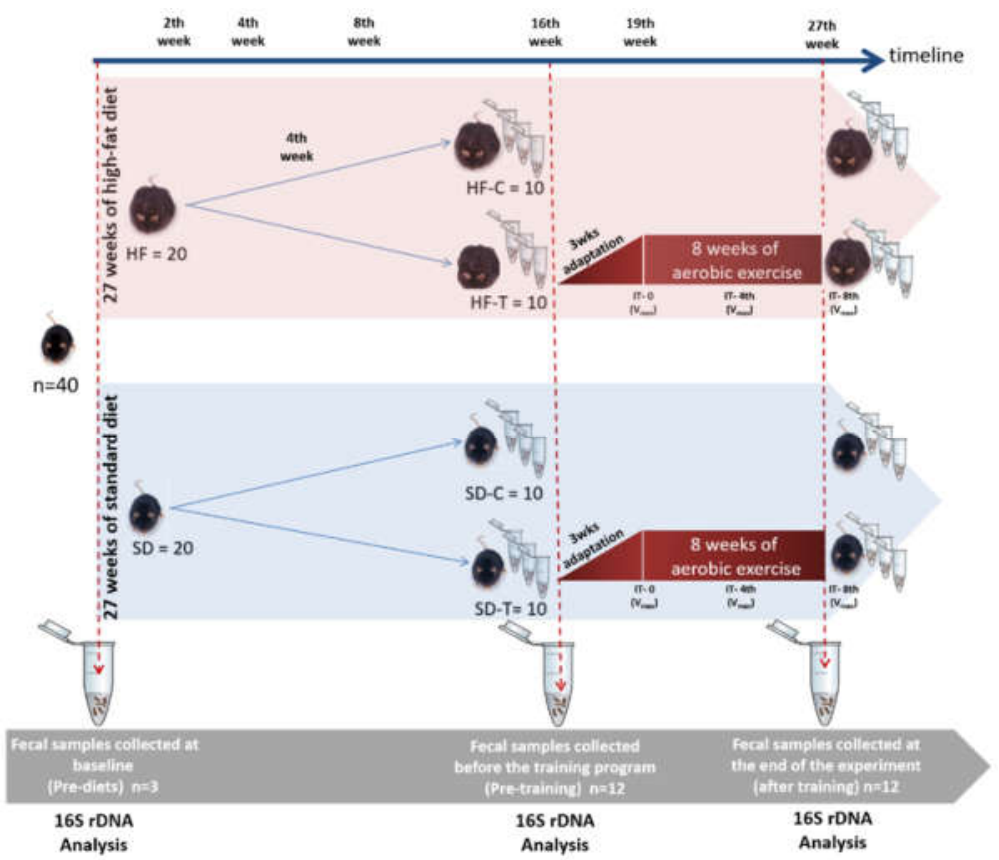

Figure 1. Experimental design. C57BL6 male mice $(n=40)$ were divided into two groups: high fat $(\mathrm{HF})$ and standard diet (SD). Afterward, animals were subdivided into four groups: standard diet control (SD-C, $n=10)$; standard diet trained (SD-T, $n=10)$; high-fat control (HF-C, $n=1$ ); and high-fat trained (HF-T, $n=10$ ). IT-0 (incremental test before training), IT-4 (IT at the 4 th week of training), IT- 8 (IT at the 8th week of training). Fecal samples were collected in the following periods: pre-diet, before training, and after 8 weeks of training.

\subsection{Analysis of $16 S \mathrm{rDNA}$ Sequences}

All of the $16 \mathrm{~S}$ rDNA amplicons were processed by the quantitative insights into microbial ecology (QIIME) pipeline version 1.9.0-dev. [33]. The sequences obtained were filtered for quality control and grouped into operational taxonomic units (OTUs) using 97\% similarity. Briefly, the phylotypes were grouped at the species level using UCLUST as standard parameter [34]. Samples were used for the rarefaction analysis to determine alpha diversity. The principal coordinate analysis (PCoA) of unweighted UniFrac distances was calculated and compared between the period of pre-diet (absolute control), before training (SD-C and HF-C) and after training (SD-T and HF-T) to observe the similarities in the composition of the gut microbiota. Beta diversity and PCoA analysis were also calculated within the QIIME using unweighted UniFrac distances.

\subsection{Statistical Analysis}

Statistical differences of the samples were based on a measure of distance in the PCoA plot using the analysis of similarities (ANOSIM) by permutation of group membership with 999 replicates [35]. The statistical test of the taxonomic differences between the samples was calculated by the ANOVA variance analysis with the Tukey test to calculate the confidence interval (nominal coverage of 99\%) and comparison of the means, using the Statistical Analysis of Metagenomic Profiles (STAMP) software version 2.0.0 [36]. Statistical analysis of alpha diversity and other physiological variables (e.g., body weight, food intake, adiposity index, and running performance) were performed on GraphPad Prism 5.00 (ANOVA with Tukey-Kramer post-hoc test considering $p<0.05$ ). The experiment was conducted just once. 


\subsection{Accession Number}

The Illumina MiSeq read data for all samples, which have been deposited in the National Center for Biotechnology Information (NCBI) Sequence Read Archive database, project number: SRP130142.

\section{Results}

\subsection{Effect of High-Fat Diet and Low-to-Moderate Exercise on Body Weight, Adiposity, Food Intake and Calorie Consumption}

During the diet administration (when compared to the first and last week: pre-diet vs. 27 weeks), a significant weight gain was observed in the animals fed with HFD when compared to those fed with SD (mean difference of $36.7 \%$ of the weight, $p<0.0001$ ). The control groups SD-C and HF-C presented a significant difference from the second month of ongoing diet $(p<0.0001)$, being represented by the letter " $a$ " (Figure 2A). The trained groups (SD-T and HF-T) had significant differences from the third month ( $p<0.01)$, being represented by the letter " $b$ " (Figure 2A). High-fat groups had higher levels of adipose tissue compared to standard diet groups (Figure 2B). Interestingly, there were no significant changes due to physical training at a low-to-moderate intensity ( $50 \%$ of IT- $\left.\mathrm{V}_{\max }\right)$. There were significant differences in the food intake between the control groups SD-C and SD-T vs. HF-C and HF-T before training $(p<0.0001)$ and between the trained groups SD-C and SD-T vs. HF-C and HF-T after training $(p<0.01)$ (Figure 3A). Also, no difference was observed in caloric intake (kcal) from all experimental groups (Figure $3 \mathrm{~B}$ ). Thus, the caloric intake in all groups was similar to their respective compositions (HFD with $60 \%$ lipids and SD with $10 \%$ lipids).

\section{A}

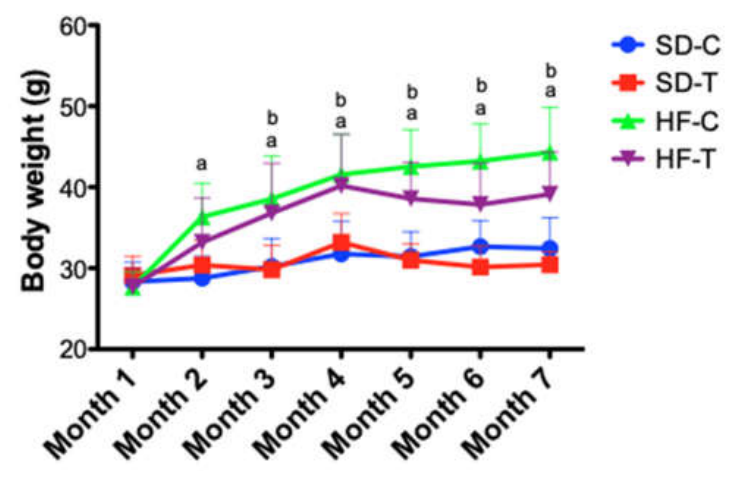

B

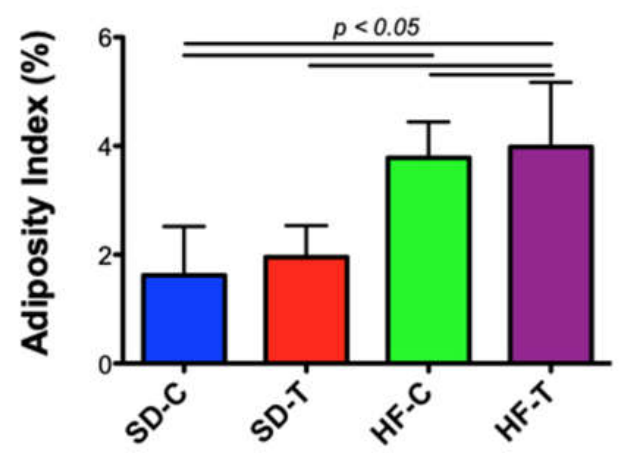

Figure 2. Effects of diets and exercise on body weight and adiposity index. (A) Two-way ANOVA was used to verify differences of body weight over eight weeks. The "a" means difference between control groups (SD-C vs. HF-C) at the same time. The " $\mathrm{b}$ " indicates differences between the trained groups (SD-T vs. HF-T). There was interaction between "diet $x$ exercise", $p<0.001, F=3.31$. (B) Significant differences between control groups compared to exercise groups ( $p<0.05$, represented as a line). 
A

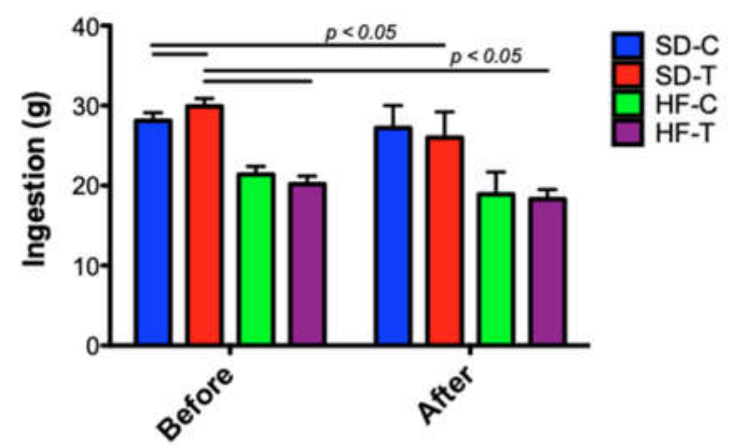

B

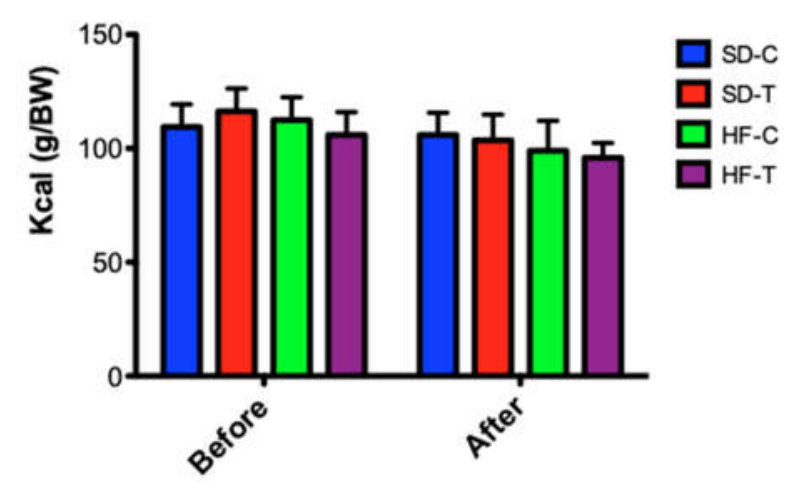

Figure 3. Food intake and kilocalories ingested by all animal groups. (A) Significant differences of food intake between the control groups when compared to respectively exercised groups (diet vs. training, $p>0.05$, diet, $p<0.05$, and training, $p<0.05$ ). (B) There is no difference in caloric intake between groups.

\subsection{Effect of Low-to-Moderate Aerobic Exercise on Aerobic Power $\left(V_{\max 2}\right)$}

After eight weeks of treadmill running at a low-to-moderate intensity, corresponding to $50 \%$ of the IT- $\mathrm{V}_{\max }$, two-way ANOVA revealed that no significant difference was observed in interaction (diet vs. exercise, $p>0.05$ ) but with differences between moments (before vs. after), $p=0.04$, and groups $(p<0.001$, Figure 4).

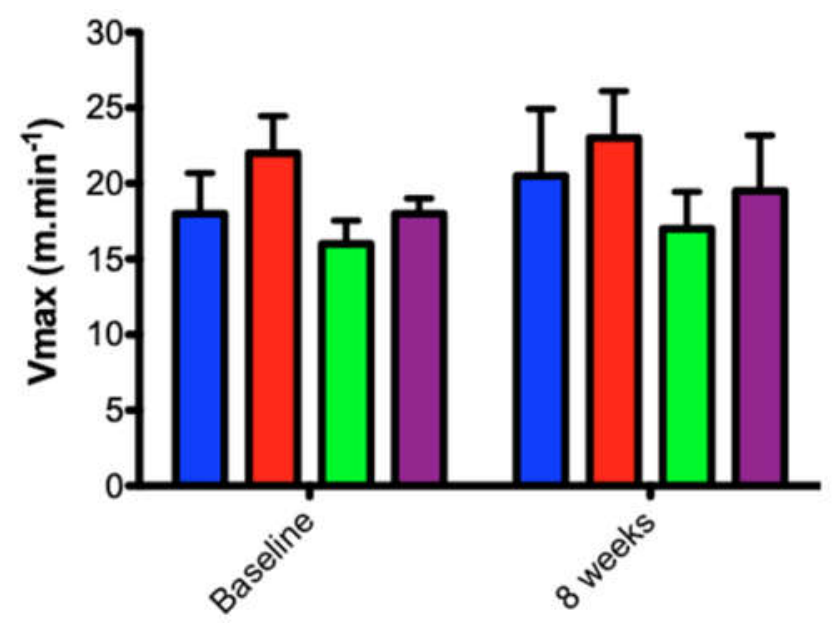

Figure 4. Incremental test. Two-way ANOVA revealed no significant differences between control and exercised groups (interaction, $p>0.05$, diet, $p<0.05$, and training, $p<0.05$ ). 


\subsection{Characterization of the Bacterial Community by Gut Microbiota Profiling}

After quality filtering, due to the expected value discrepancy between regions during sequencing, it was necessary to normalize the samples, delimiting the number of minimum sequences for maximum utilization. Thus, analyses of $\alpha$ - and $\beta$-diversity were performed with samples that obtained a number greater than or equal to 113,854 sequences for each sample. Therefore, one of the biological replicates did not contain the minimum number of sequences mentioned above, which led to its exclusion from $\alpha$ - and $\beta$-diversity analysis.

In this context, bacterial diversity was assessed by the rarefaction measure of observed species against the number of sequences per sample. The $\alpha$-diversity analysis (Shannon-Wiener index) revealed that the OTU richness in pre-diet fecal samples is more species-rich than those found both in samples after four months of diet application, as well as after the training intervention (Figure 5).

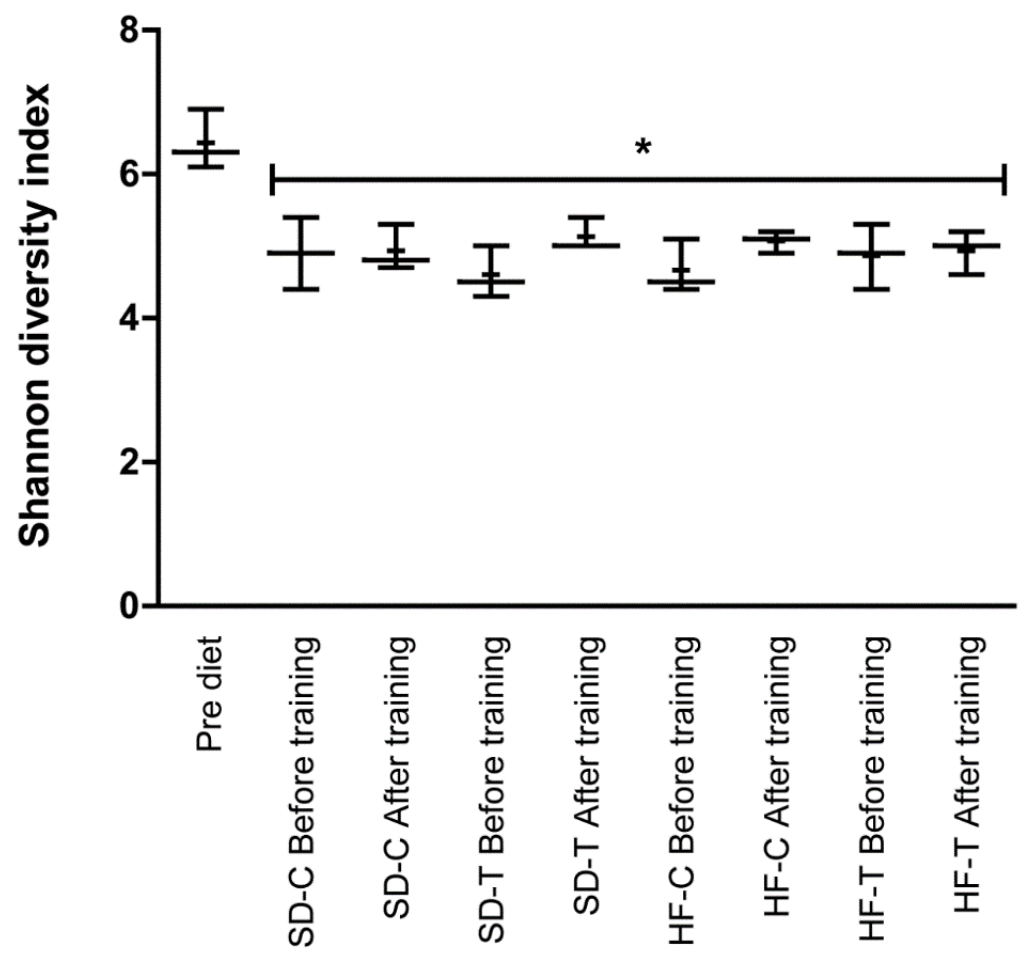

\section{Experimental groups}

Figure 5. Bacterial alpha diversity. Comparison of alpha diversity estimation of the 16S rRNA gene libraries at $97 \%$ similarity from the sequencing analysis (ANOVA with Tukey-Kramer post-hoc test).

\subsection{Principal Coordinates Analysis (PCoA)}

Principal coordinates analysis (PCoA) of unweighted UniFrac distances aimed to compare the effect of diet and exercise on the bacterial community of all samples. Unweighted UniFrac (PCoA) analysis of the fecal samples presented a higher similarity of the bacterial composition due to the application of both diet interventions (HF and SD) (Figure 6). Here, animals that were fed with HD (before and after training) are plotted in the lower quadrant, and samples fed with SD (before and after training) are plotted in the upper quadrant (Figure 6). These data indicate that the diet application led to a different bacterial composition between the two diets (HFD and SD diets), both after four months as well as after low-to-moderate exercise $(\mathrm{R}=0.53, p<0.001)$. The time difference between periods (pre-diet vs. before and after training) also played a role in the distinct bacterial community compositions for these different groups. Accordingly, eight weeks of exercise training performed at 
a low-to-moderate intensity (50\% of maximal velocity) did not cause reliable modifications in the composition of the bacterial community $(\mathrm{R}=-0.048, p=0.79)$.

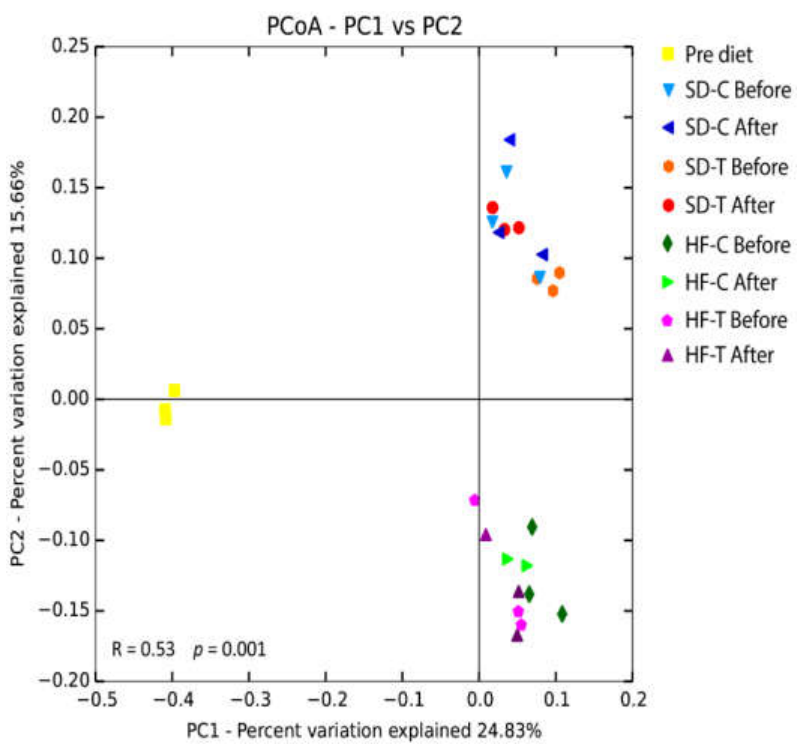

Figure 6. Effect of diets on the bacterial community. The PCoA of unweighted UniFrac distances was generated with the sequences obtained by the samples in the pre-diet (absolute control), and before and after training periods. The ANOSIM similarity analysis results show significant alteration of the gut microbiota by the diets.

\subsection{Effects of Low-to-Moderate Exercise on Bacterial Diversity}

Concerning the modulation in the abundance of bacterial genera due to low-to-moderate exercise (95\% confidence interval), Proteus and Vagococcus were shown to be altered (Figure 7). The comparison between all experimental groups indicated that those submitted to high-fat diet obtained greater expressions of the genera Proteus (HF-C and HF-T), especially the HF-C group (Figure 7A). These data indicate that exercise played a role in reducing Proteus expression. The standard dietary groups (SD-C and SD-T) presented lower expression of Proteus. Finally, the exercise protocol (SD-T and HF-T) was relevant in the expression of the genus Vagococcus (Figure 7B). Besides, when compared to the trained groups (SD-T and HF-T), it can be observed that the high-fat diet decreased the expression of Vagococcus.
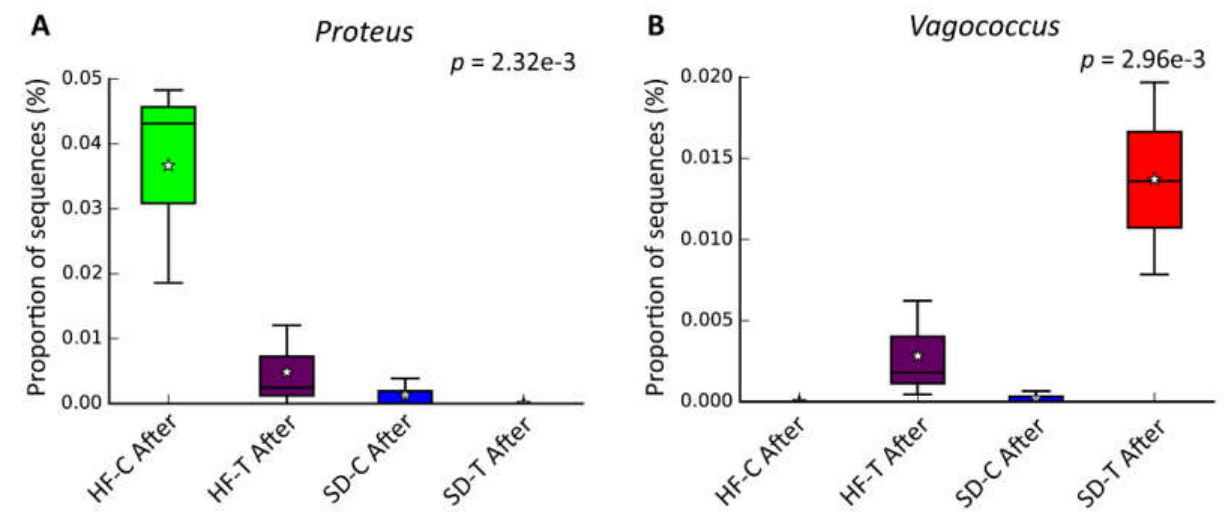

Figure 7. Effect of exercise training on bacterial genera profile. (A) Proteus; (B) Vagococcus. White star represents the average of each group, and the median value is shown as a line within the box. $(p<0.01$ was considered as statistical significance, ANOVA with Tukey-Kramer post-hoc test.) 


\section{Discussion}

Throughout life, the gut microbiota is significantly modulated by several environmental and host-related factors [11]. In this context, dietary intervention is one of the most potent factors in shaping the gut bacterial community $[8,15,35]$. Studies have shown that a high-fat diet (HFD) negatively modulates the diversity and composition of bacteria in mice $[35,36]$. These changes within the gut microbiota were observed over eight, 12 and 16 weeks [36]. In accordance with the literature, in our study, 8 weeks of induction by HFD was accompanied by a significant increase in body weight and adiposity index (Figure 2), resulting in a lower bacterial diversity (Figure 6).

Some studies discuss the effect of exercise, where exercise solely does not seem to be effective for weight reduction [37,38], especially in obese models (e.g., Zucker rats) [39]. Exercise has been partially beneficial in improving health in rodents consuming an HFD, whereas incorporating a "better" diet can ameliorate the metabolism and general health [38]. However, in our study, the protocol of eight weeks of low-to-moderate exercise was not able to reduce the weight gain and adiposity index among the HFD groups (HF-T vs. HF-C). Thus, these groups presented an improvement in weight and adiposity when compared to the student diets groups (Figure 2). Moreover, it was observed that HFD tends to alter food satiety, also de-affecting the process of appetite control [40,41]. According to our data, the ingestion (in grams) of animals fed with HFD was lower, when compared to the animals fed SD. However, although the caloric amount of HFD is higher than SD, the caloric intake of all groups was similar, with no statistically significant difference between them. Thus, the increase in weight gain (Figure 2) of the HFD groups may have been caused by different dietary macronutrient metabolism [42].

Following the incremental test of maximal velocity $\left(\mathrm{IT}-\mathrm{V}_{\max }\right)$ (Figure 4$)$, the HFD may have influenced the physical performance of the respective groups. This was verified by a better performance of the SD-C vs. HF-C $(p<0.05)$ and SD-T vs. HFD groups before training $(p<0.01)$. Also, there was no statistical difference between HF-T and HF-C after training. This adverse effect on running performance has also been seen in other studies [43,44]. The exposure to high-fat diets is associated with faster accumulation of blood lactate [43], reduction in lipolytic signaling pathways [45], disturbed glucose metabolism [46] and impairment of skeletal muscle mitochondrial function [44]. In our study, it is possible that these physiological aspects may have influenced the performance of the IT- $V_{\max }$ before the training period. In agreement with this, it was observed that the SD-C group also had a better performance before and after training, when compared to the HF-C group $(p<0.05)$ (Figure 4$)$. Similarly, the SD-T group also continued to have an improvement in aerobic power compared to HFD groups after training $(p<0.05)$. However, beneficial results have been observed in athletes that consume an ideal dietary fatty acid composition, and it appears that induction by HFD is required for several months $[47,48]$.

Regardless of diet, better cardiorespiratory fitness has been recently correlated with increased gut microbial diversity [49]. In this sense, animals fed HFD did not improve performance with training (Figure 4). However, other studies with different intensities such as high-intensity interval training (HIIT) [20] and endurance exercise showed that they have a different role in microbial diversity [50]. Our results showed an increase of the Proteus genus in high-fat diet groups (Figure 7A). It is noteworthy that the HF-T group obtained a lower expression of Proteus compared to the control (HF-C). This effect of exercise on the lower abundance of Proteus was also visualized in the standard diet group that trained (SD-T vs. SD-C).

Proteus genus species have been commonly observed in the intestinal tract and considered as human opportunistic pathogens, where these bacteria are known to cause infection during impaired immunity [51]. Besides, elevated levels of Proteus mirabilis abundance have been associated with several inflammatory processes including inflammatory bowel disease and inflammatory arthritis and rheumatoid arthritis [52,53]. Furthermore, an increased P. mirabilis abundance was observed in rats that underwent a high-fat diet, also being significantly correlated with triglyceride levels, and leptin and insulin concentration, indicating that this bacterium may be associated with low-grade 
inflammation linked to obesity [54]. Some Proteus bacteria have been related to lipopolysaccharide (LPS) biosynthesis [55]. It is well known that elevated levels of LPSs are linked to inflammatory reactions in obesity [56]. Thus, as observed in our study, the lower abundance of the Proteus genus after prolonged aerobic exercise may be a positive indicator and control from low-grade inflammation associated with obesity.

An increase in the abundance of Vagococcus genera was also observed in the trained groups (Figure 7B). This genus belongs to the Enterococcaceae family, well known for its bacteria that synthetize lactic acid as their primary metabolic end product [51,52]. Despite its unclear mechanism, lactic acid bacteria are known to have an anti-obesity effect [53,54]. Species such as Lactobacillus gasseri and Lactobacillus delbrueckii are reported for their effect on cholesterol metabolism [55]. Also, the administration of L. gasseri as a probiotic supplement was reported to reduce adiposity and body weight in obese adults [55]. The Enterococcaceae family was also associated with a low-fat diet (10\% fat) in a study that investigated the effect of a high-fat diet and genetically induced obesity on the intestinal microbiome and metabolome of a mouse model for colorectal cancer [56]. Despite the increase of Vagococcus genera after training, more studies are necessary to further understand the anti-obesity effect of these bacteria.

Although exercise has played a role in reducing Proteus and increasing Vagococcus, these data reveal that exercise with low-to-moderate intensity has a limited effect on gut microbiota. It is important to note that the time of only high-fat diet induction (16 weeks) was twice the time of exercise application (8 weeks). In this way, more studies with different protocols of physical training in contrast to the high-fat diet become necessary. Another factor that may have influenced the results is the intensity applied in the present training protocol. Our results show for the first time that low-to-moderate intensity was not able to counteract the effects of prolonged HFD induction. However, the experiments from our study were conducted just once, being a limiting point to be considered in this study.

\section{Conclusions}

The present study demonstrated that gut microbiota diversity was reduced by diet in all experimental groups. Despite being the first study to apply a low-to-moderate aerobic training protocol, this stimulus presented a reduced effect on gut microbiota shaping when contrasted with the high-fat diet used to induce obesity. However, other training variables such as intensity, volume and even other types of exercise stimuli must be explored, particularly when applied to pathologic models such as obesity. As recently reported by Mitchell et al. [57], rigorous dietary control in larger samples is essential to further identify the influence and mechanisms of exercise on the gut microbiota, and how these stimuli may be extended to novel clinical treatments of metabolic diseases such as obesity.

Author Contributions: F.M.R.: Conceived the study and carried out all experimental procedures and drafted the manuscript, C.F.A.R.: carried out the bioinformatics design and revised the manuscript, A.C.M.G.: carried out the in vivo and in vitro experiment on the samples and revised the manuscript. A.P.C.: carried out the bioinformatics design, performed the statistical analysis and revised the manuscript, J.A.A.: participated in the study design, revised all experimental procedures, performed the statistical analysis and revised the manuscript, O.L.F.: participated in the study design, revised all experimental procedures and drafted and revised manuscript. B.A.P.: participated in the study design, revised all experimental procedures and drafted and revised manuscript. All authors read and approved the final manuscript.

Acknowledgments: This research was funded by CNPq (protocol number: 152817/2016-6), CAPES, FAPDF, UDF, and FUNDECT.

Conflicts of Interest: The authors declare no conflict of interest.

\section{References}

1. Parikh, N.I.; Pencina, M.J.; Wang, T.J.; Lanier, K.J.; Fox, C.S.; D'Agostino, R.B.; Vasan, R.S. Increasing trends in incidence of overweight and obesity over 5 decades. Am. J. Med. 2007, 120, 242-250. [CrossRef]

2. World Health Organization. Obesity and Overweight_Fact Sheet; World Health Organization: Geneva, Switzerland, 2017. 
3. Lehnert, T.; Sonntag, D.; Konnopka, A.; Riedel-Heller, S.; Konig, H.H. Economic costs of overweight and obesity. Best Pract. Res. Clin. Endocrinol. Metab. 2013, 27, 105-115. [CrossRef]

4. The Global BMI Mortality Collaboration; Di Angelantonio, E.; Bhupathiraju, S.N.; Wormser, D.; Gao, P.; Kaptoge, S.; Berrington de Gonzalez, A.; Cairns, B.J.; Huxley, R.; Jackson, C.L.; et al. Body-mass index and all-cause mortality: Individual-participant-data meta-analysis of 239 prospective studies in four continents. Lancet 2016, 388, 776-786. [CrossRef]

5. Apovian, C.M.; Riffenburg, K.M. Perspectives on the global obesity epidemic. Curr. Opin. Endocrinol. Diabetes Obes. 2017, 24, 307-309. [CrossRef]

6. Turnbaugh, P.J.; Hamady, M.; Yatsunenko, T.; Cantarel, B.L.; Duncan, A.; Ley, R.E.; Sogin, M.L.; Jones, W.J.; Roe, B.A.; Affourtit, J.P.; et al. A core gut microbiome in obese and lean twins. Nature 2009, 457, 480-484. [CrossRef]

7. Cano, P.G.; Santacruz, A.; Trejo, F.M.; Sanz, Y. Bifidobacterium CECT 7765 improves metabolic and immunological alterations associated with obesity in high-fat diet-fed mice. Obesity 2013, 21, 2310-2321. [CrossRef]

8. Li, J.; Riaz Rajoka, M.S.; Shao, D.; Jiang, C.; Jin, M.; Huang, Q.; Yang, H.; Shi, J. Strategies to increase the efficacy of using gut microbiota for the modulation of obesity. Obes. Rev. Off. J. Int. Assoc. Study Obes. 2017, 18, 1260-1271. [CrossRef]

9. Rial, S.A.; Karelis, A.D.; Bergeron, K.F.; Mounier, C. Gut Microbiota and Metabolic Health: The Potential Beneficial Effects of a Medium Chain Triglyceride Diet in Obese Individuals. Nutrients 2016, 8, 281. [CrossRef]

10. Bermon, S.; Petriz, B.; Kajeniene, A.; Prestes, J.; Castell, L.; Franco, O.L. The microbiota: An exercise immunology perspective. Exerc. Immunol. Rev. 2015, 21, 70-79.

11. Org, E.; Mehrabian, M.; Lusis, A.J. Unraveling the environmental and genetic interactions in atherosclerosis: Central role of the gut microbiota. Atherosclerosis 2015, 241, 387-399. [CrossRef]

12. Pickard, J.M.; Zeng, M.Y.; Caruso, R.; Nunez, G. Gut microbiota: Role in pathogen colonization, immune responses, and inflammatory disease. Immunol. Rev. 2017, 279, 70-89. [CrossRef] [PubMed]

13. Zhang, X.; Zhao, Y.; Xu, J.; Xue, Z.; Zhang, M.; Pang, X.; Zhang, X.; Zhao, L. Modulation of gut microbiota by berberine and metformin during the treatment of high-fat diet-induced obesity in rats. Sci. Rep. 2015, 5, 14405. [CrossRef] [PubMed]

14. Houghton, D.; Stewart, C.J.; Day, C.P.; Trenell, M. Gut Microbiota and Lifestyle Interventions in NAFLD. Int. J. Mol. Sci. 2016, 17, 447. [CrossRef]

15. Collins, K.H.; Paul, H.A.; Hart, D.A.; Reimer, R.A.; Smith, I.C.; Rios, J.L.; Seerattan, R.A.; Herzog, W. A High-Fat High-Sucrose Diet Rapidly Alters Muscle Integrity, Inflammation and Gut Microbiota in Male Rats. Sci. Rep. 2016, 6, 37278. [CrossRef] [PubMed]

16. Malikowski, T.; Khanna, S.; Pardi, D.S. Fecal microbiota transplantation for gastrointestinal disorders. Curr. Opin. Gastroenterol. 2017, 33, 8-13. [CrossRef] [PubMed]

17. Petriz, B.A.; Castro, A.P.; Almeida, J.A.; Gomes, C.P.; Fernandes, G.R.; Kruger, R.H.; Pereira, R.W.; Franco, O.L. Exercise induction of gut microbiota modifications in obese, non-obese and hypertensive rats. BMC Genom. 2014, 15, 511. [CrossRef] [PubMed]

18. Bressa, C.; Bailen-Andrino, M.; Perez-Santiago, J.; Gonzalez-Soltero, R.; Perez, M.; Montalvo-Lominchar, M.G.; Mate-Munoz, J.L.; Dominguez, R.; Moreno, D.; Larrosa, M. Differences in gut microbiota profile between women with active lifestyle and sedentary women. PLoS ONE 2017, 12, e0171352. [CrossRef]

19. Yang, Y.; Shi, Y.; Wiklund, P.; Tan, X.; Wu, N.; Zhang, X.; Tikkanen, O.; Zhang, C.; Munukka, E.; Cheng, S. The Association between Cardiorespiratory Fitness and Gut Microbiota Composition in Premenopausal Women. Nutrients 2017, 9, 792. [CrossRef]

20. Denou, E.; Marcinko, K.; Surette, M.G.; Steinberg, G.R.; Schertzer, J.D. High-intensity exercise training increases the diversity and metabolic capacity of the mouse distal gut microbiota during diet-induced obesity. Am. J. Physiol. Endocrinol. Metab. 2016, 310, E982-E993. [CrossRef]

21. Liu, Z.; Liu, H.Y.; Zhou, H.; Zhan, Q.; Lai, W.; Zeng, Q.; Ren, H.; Xu, D. Moderate-Intensity Exercise Affects Gut Microbiome Composition and Influences Cardiac Function in Myocardial Infarction Mice. Front. Microbiol. 2017, 8, 1687. [CrossRef]

22. Lamoureux, E.V.; Grandy, S.A.; Langille, M.G.I. Moderate Exercise Has Limited but Distinguishable Effects on the Mouse Microbiome. MSystems 2017, 2, e00006-17. [CrossRef] [PubMed] 
23. Allen, J.M.; Berg Miller, M.E.; Pence, B.D.; Whitlock, K.; Nehra, V.; Gaskins, H.R.; White, B.A.; Fryer, J.D.; Woods, J.A. Voluntary and forced exercise differentially alters the gut microbiome in C57BL/6J mice. J. Appl. Physiol. 2015, 118, 1059-1066. [CrossRef] [PubMed]

24. Monda, V.; Villano, I.; Messina, A.; Valenzano, A.; Esposito, T.; Moscatelli, F.; Viggiano, A.; Cibelli, G.; Chieffi, S.; Monda, M.; et al. Exercise Modifies the Gut Microbiota with Positive Health Effects. Oxid. Med. Cell. Longev. 2017, 2017, 3831972. [CrossRef] [PubMed]

25. Hsu, Y.J.; Huang, W.C.; Lin, J.S.; Chen, Y.M.; Ho, S.T.; Huang, C.C.; Tung, Y.T. Kefir Supplementation Modifies Gut Microbiota Composition, Reduces Physical Fatigue, and Improves Exercise Performance in Mice. Nutrients 2018, 10, 862. [CrossRef] [PubMed]

26. Welly, R.J.; Liu, T.W.; Zidon, T.M.; Rowles, J.L., 3rd; Park, Y.M.; Smith, T.N.; Swanson, K.S.; Padilla, J.; Vieira-Potter, V.J. Comparison of Diet versus Exercise on Metabolic Function and Gut Microbiota in Obese Rats. Med. Sci. Sports Exerc. 2016, 48, 1688-1698. [CrossRef] [PubMed]

27. Evans, C.C.; LePard, K.J.; Kwak, J.W.; Stancukas, M.C.; Laskowski, S.; Dougherty, J.; Moulton, L.; Glawe, A.; Wang, Y.; Leone, V.; et al. Exercise prevents weight gain and alters the gut microbiota in a mouse model of high fat diet-induced obesity. PLoS ONE 2014, 9, e92193. [CrossRef] [PubMed]

28. Hildebrand, F.; Nguyen, T.L.; Brinkman, B.; Yunta, R.G.; Cauwe, B.; Vandenabeele, P.; Liston, A.; Raes, J. Inflammation-associated enterotypes, host genotype, cage and inter-individual effects drive gut microbiota variation in common laboratory mice. Genome Biol. 2013, 14, R4. [CrossRef] [PubMed]

29. Ramdhave, A.S.; Ojha, S.; Nandave, M. Energy intake correlates with the levels of fatty acid synthase and insulin-like growth factor-1 in male and female C57BL/6 mice. Am. J. Transl. Res. 2017, 9, 830-844.

30. Taylor, B.A.; Phillips, S.J. Detection of obesity QTLs on mouse chromosomes 1 and 7 by selective DNA pooling. Genomics 1996, 34, 389-398. [CrossRef]

31. Almeida, J.A.; Petriz Bde, A.; da Costa Gomes, C.P.; Pereira, R.W.; Franco, O.L. Assessment of maximal lactate steady state during treadmill exercise in SHR. BMC Res. Notes 2012, 5, 661. [CrossRef]

32. Almeida, J.A.; Petriz, B.A.; Gomes, C.P.; Rocha, L.A.; Pereira, R.W.; Franco, O.L. Determination of the maximal lactate steady state in obese Zucker rats. Int. J. Sports Med. 2013, 34, 214-217. [CrossRef] [PubMed]

33. Caporaso, J.G.; Kuczynski, J.; Stombaugh, J.; Bittinger, K.; Bushman, F.D.; Costello, E.K.; Fierer, N.; Pena, A.G.; Goodrich, J.K.; Gordon, J.I.; et al. QIIME allows analysis of high-throughput community sequencing data. Nat. Methods 2010, 7, 335-336. [CrossRef] [PubMed]

34. Edgar, R.C. Search and clustering orders of magnitude faster than BLAST. Bioinformatics 2010, 26, $2460-2461$. [CrossRef] [PubMed]

35. Daniel, H.; Gholami, A.M.; Berry, D.; Desmarchelier, C.; Hahne, H.; Loh, G.; Mondot, S.; Lepage, P.; Rothballer, M.; Walker, A.; et al. High-fat diet alters gut microbiota physiology in mice. ISME J. 2014, 8 , 295-308. [CrossRef] [PubMed]

36. Guo, X.; Li, J.; Tang, R.; Zhang, G.; Zeng, H.; Wood, R.J.; Liu, Z. High Fat Diet Alters Gut Microbiota and the Expression of Paneth Cell-Antimicrobial Peptides Preceding Changes of Circulating Inflammatory Cytokines. Mediators Inflamm. 2017, 2017, 9474896. [CrossRef] [PubMed]

37. Clark, J.E. Diet, exercise or diet with exercise: Comparing the effectiveness of treatment options for weight-loss and changes in fitness for adults (18-65 years old) who are overfat, or obese; systematic review and meta-analysis. J. Diabetes Metab. Disord. 2015, 14, 31. [CrossRef] [PubMed]

38. Hatzidis, A.; Hicks, J.A.; Gelineau, R.R.; Arruda, N.L.; Monteiro De Pina, I.; O'Connell, K.E.; Seggio, J.A. Removal of a high-fat diet, but not voluntary exercise, reverses obesity and diabetic-like symptoms in male C57BL/6J mice. Hormones 2017, 16, 62-74. [CrossRef]

39. Almeida, J.A.; Petriz, B.A.; Gomes, C.P.; Araujo, R.C.; Pereira, R.W.; Franco, O.L. Exercise training at MLSS decreases weight gain and increases aerobic capacity in obese Zucker rats. Int J. Sports Med. 2014, 35, 199-202. [CrossRef]

40. Kentish, S.J.; Wittert, G.A.; Blackshaw, L.A.; Page, A.J. A chronic high fat diet alters the homologous and heterologous control of appetite regulating peptide receptor expression. Peptides 2013, 46, 150-158. [CrossRef]

41. Covasa, M.; Ritter, R.C. Rats maintained on high-fat diets exhibit reduced satiety in response to CCK and bombesin. Peptides 1998, 19, 1407-1415. [CrossRef]

42. Abete, I.; Astrup, A.; Martinez, J.A.; Thorsdottir, I.; Zulet, M.A. Obesity and the metabolic syndrome: Role of different dietary macronutrient distribution patterns and specific nutritional components on weight loss and maintenance. Nutr. Rev. 2010, 68, 214-231. [CrossRef] 
43. Chen, C.N.; Liao, Y.H.; Lin, S.Y.; Yu, J.X.; Li, Z.J.; Lin, Y.C.; Chang, G.J.; Lin, C.H.; Wong, A.M. Diet-induced obesity accelerates blood lactate accumulation of rats in response to incremental exercise to maximum. Am. J. Physiol. Regul. Integr. Comp. Physiol. 2017, 313, R601-R607. [CrossRef] [PubMed]

44. Takada, S.; Kinugawa, S.; Matsushima, S.; Takemoto, D.; Furihata, T.; Mizushima, W.; Fukushima, A.; Yokota, T.; Ono, Y.; Shibata, H.; et al. Sesamin prevents decline in exercise capacity and impairment of skeletal muscle mitochondrial function in mice with high-fat diet-induced diabetes. Exp. Physiol. 2015, 100, 1319-1330. [CrossRef] [PubMed]

45. Chen, N.; Cheng, J.; Zhou, L.; Lei, T.; Chen, L.; Shen, Q.; Qin, L.; Wan, Z. Effects of treadmill running and rutin on lipolytic signaling pathways and TRPV4 protein expression in the adipose tissue of diet-induced obese mice. J. Physiol. Biochem. 2015, 71, 733-742. [CrossRef] [PubMed]

46. Mardare, C.; Kruger, K.; Liebisch, G.; Seimetz, M.; Couturier, A.; Ringseis, R.; Wilhelm, J.; Weissmann, N.; Eder, K.; Mooren, F.C. Endurance and Resistance Training Affect High Fat Diet-Induced Increase of Ceramides, Inflammasome Expression, and Systemic Inflammation in Mice. J. Diabetes Res. 2016, 2016, 4536470. [CrossRef]

47. Chang, C.K.; Borer, K.; Lin, P.J. Low-Carbohydrate-High-Fat Diet: Can it Help Exercise Performance? J. Hum. Kinet. 2017, 56, 81-92. [CrossRef] [PubMed]

48. Lindseth, I. Methodological issues question the validity of observed performance impairment of a low carbohydrate, high fat diet. J. Physiol. 2017, 595, 2989. [CrossRef]

49. Estaki, M.; Pither, J.; Baumeister, P.; Little, J.P.; Gill, S.K.; Ghosh, S.; Ahmadi-Vand, Z.; Marsden, K.R.; Gibson, D.L. Cardiorespiratory fitness as a predictor of intestinal microbial diversity and distinct metagenomic functions. Microbiome 2016, 4, 42. [CrossRef]

50. Hsu, Y.J.; Chiu, C.C.; Li, Y.P.; Huang, W.C.; Huang, Y.T.; Huang, C.C.; Chuang, H.L. Effect of intestinal microbiota on exercise performance in mice. J. Strength Cond. Res. Natl. Strength Cond. Assoc. 2015, 29, 552-558. [CrossRef]

51. Wang, L.; Cui, Y.S.; Kwon, C.S.; Lee, S.T.; Lee, J.S.; Im, W.T. Vagococcus acidifermentans sp. nov., isolated from an acidogenic fermentation bioreactor. Int. J. Syst Evol. Microbiol. 2011, 61, 1123-1126. [CrossRef]

52. Juturu, V.; Wu, J.C. Microbial production of lactic acid: The latest development. Crit. Rev. Biotechnol. 2016, 36, 967-977. [CrossRef]

53. Yonejima, Y.; Ushida, K.; Mori, Y. Effect of Lactic Acid Bacteria on Lipid Metabolism and Fat Synthesis in Mice Fed a High-fat Diet. Biosci. Microbiota Food Health 2013, 32, 51-58. [CrossRef] [PubMed]

54. Usman; Hosono, A. Bile tolerance, taurocholate deconjugation, and binding of cholesterol by Lactobacillus gasseri strains. J. Dairy Sci. 1999, 82, 243-248. [CrossRef]

55. Kadooka, Y.; Sato, M.; Imaizumi, K.; Ogawa, A.; Ikuyama, K.; Akai, Y.; Okano, M.; Kagoshima, M.; Tsuchida, T. Regulation of abdominal adiposity by probiotics (Lactobacillus gasseri SBT2055) in adults with obese tendencies in a randomized controlled trial. Eur. J. Clin. Nutr. 2010, 64, 636-643. [CrossRef] [PubMed]

56. Pfalzer, A.C.; Nesbeth, P.D.; Parnell, L.D.; Iyer, L.K.; Liu, Z.; Kane, A.V.; Chen, C.Y.; Tai, A.K.; Bowman, T.A.; Obin, M.S.; et al. Diet- and Genetically-Induced Obesity Differentially Affect the Fecal Microbiome and Metabolome in Apc1638N Mice. PLoS ONE 2015, 10, e0135758. [CrossRef]

57. Mitchell, C.M.; Davy, B.M.; Hulver, M.W.; Neilson, A.P.; Bennett, B.J.; Davy, K.P. Does Exercise Alter Gut Microbial Composition? A Systematic Review. Med. Sci. Sports Exerc. 2019, 51, 160-167. [CrossRef] [PubMed]

(C) 2019 by the authors. Licensee MDPI, Basel, Switzerland. This article is an open access article distributed under the terms and conditions of the Creative Commons Attribution (CC BY) license (http://creativecommons.org/licenses/by/4.0/). 